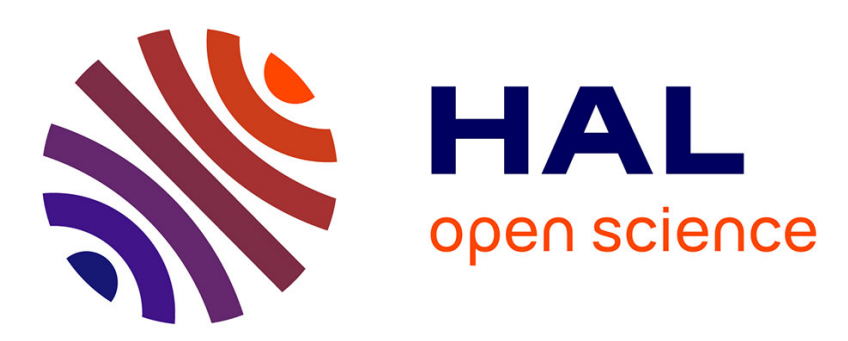

\title{
Merging sensor data from multiple temperature scenarios for vibration monitoring of civil structures
}

Etienne Balmès, Michèle Basseville, Laurent Mevel, Houssein Nasser, Frédéric Bourquin, Fabien Treyssède

\section{- To cite this version:}

Etienne Balmès, Michèle Basseville, Laurent Mevel, Houssein Nasser, Frédéric Bourquin, et al.. Merging sensor data from multiple temperature scenarios for vibration monitoring of civil structures. [Research Report] PI 1824, 2006. inria-00164852

\section{HAL Id: inria-00164852 \\ https://hal.inria.fr/inria-00164852}

Submitted on 24 Jul 2007

HAL is a multi-disciplinary open access archive for the deposit and dissemination of scientific research documents, whether they are published or not. The documents may come from teaching and research institutions in France or abroad, or from public or private research centers.
L'archive ouverte pluridisciplinaire HAL, est destinée au dépôt et à la diffusion de documents scientifiques de niveau recherche, publiés ou non, émanant des établissements d'enseignement et de recherche français ou étrangers, des laboratoires publics ou privés. 


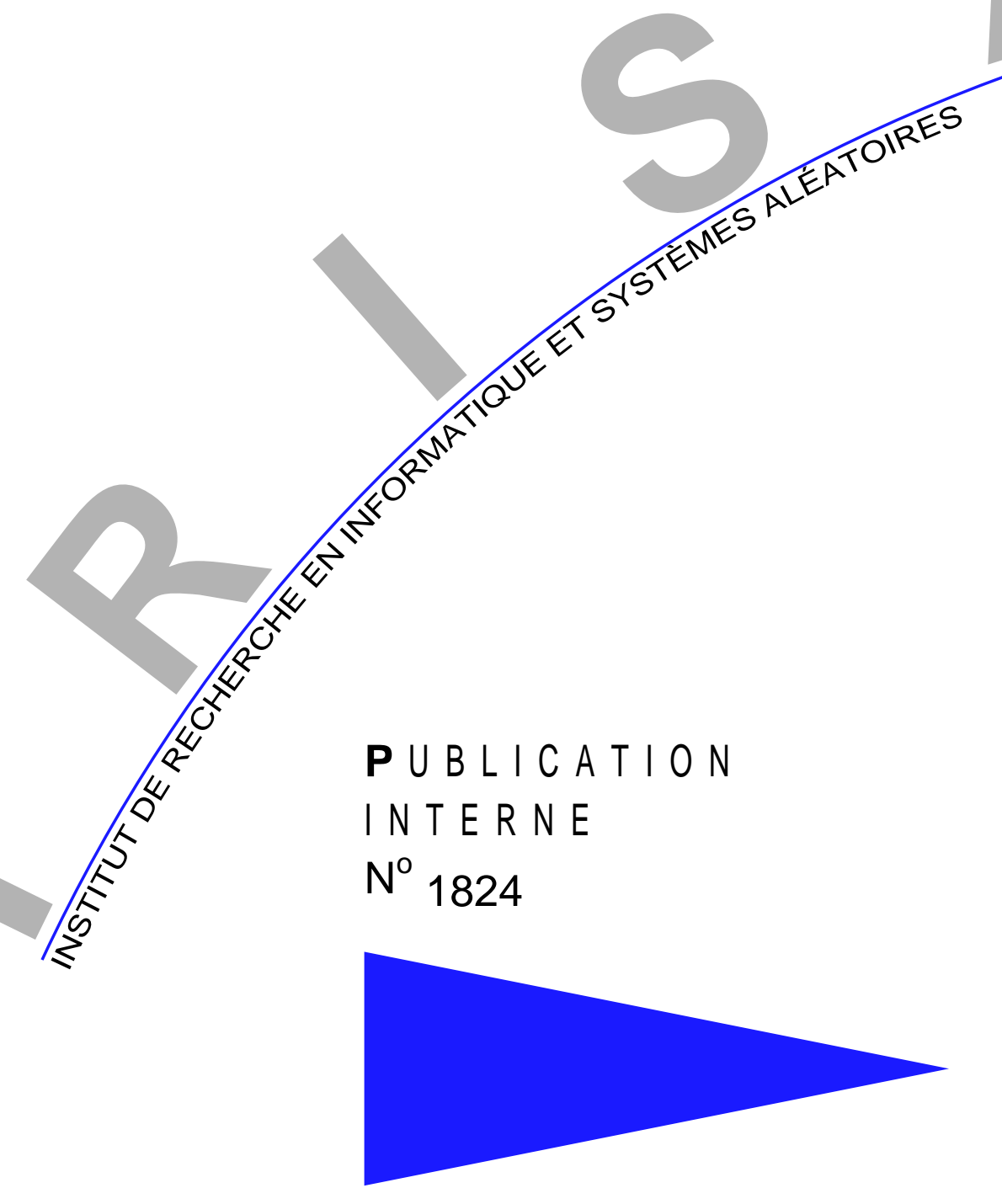

\author{
MERGING SENSOR DATA FROM MULTIPLE \\ TEMPERATURE SCENARIOS FOR VIBRATION \\ MONITORING OF CIVIL STRUCTURES
}

ÉTIENNE BALMĖS, MICHÈLE BASSEVILLE, LAURENT MEVEL, HOUSSEIN NASSER, FRÉDÉRIC BOURQUIN, FABIEN TREYSSĖDE 



\title{
Merging sensor data from multiple temperature scenarios for vibration monitoring of civil structures
}

\author{
Étienne Balmès ${ }^{*}$, Michèle Basseville, Laurent Mevel, Houssein Nasser ${ }^{* *}$, \\ Frédéric Bourquin, Fabien Treyssède ${ }^{* * *}$ \\ Systèmes numériques \\ Projet Sisthem
}

Publication interne $\mathrm{n}^{\circ} 1824-$ November $2006-21$ pages

\begin{abstract}
The ambient temperature effect may result in limitations of vibrationbased structural health monitoring (SHM) approaches for civil engineering structures. This paper addresses the issue of discriminating changes in modal parameters due to damages and changes in modal parameters due to temperature effects. A non parametric damage detection algorithm is proposed, which only assumes that several datasets are recorded on the safe structure at different and unknown temperatures, and smoothes out the temperature effect using an averaging operation.
\end{abstract}

Key-words: Subspace identification, damage detection, sensor data fusion, vibration-based monitoring, civil engineering structures, temperature effect.

(Résumé : tsvp)

This work was carried out within the project no 2003-54 CONSTRUCTIF, coordinated by IRISA, in the framework of the French Computer and Security program (ACI S\&I).

A preliminary version of this paper was presented at the 3rd European Workshop on Structural Health Monitoring, Granada, S., July 2006.

* Lmssmat, Ecole Centrale Paris, Grande Voie des Vignes, 92295 Châtenay-Malabry Cedex, F.

** IRISA - Michele.Basseville, Laurent.Mevel, Houssein.Nasser@irisa.fr. M.B. is also with CNRs, L.M. with InRIA, H.N. with Rennes 1 University.

*** LCPC, Division for Metrology and Instrumentation.

F.B.: 58, bd Lefebvre, 75732 Paris Cedex 15, F.

F.T.: Route de Bouaye, BP 4129, 44341 Bouguenais Cedex, F.

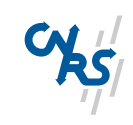

Centre National de la Recherche Scientifique (UMR 6074) Université de Rennes 1 - Insa de Rennes
Institut National de Recherche en Informatique et en Automatique - unité de recherche de Rennes 


\section{Fusion de données provenant de scenarii de température multiples pour la surveillance vibratoire de structures de génie civil}

Résumé : L'effet de la température ambiante limite l'efficacité des méthodes de surveillance d'intégrité de structures basées sur leurs vibrations. Cet article étudie la discrimination entre changements de paramètres modaux dus aux endommagements et changements de paramètres modaux dus aux effets de température. Un algorithme non-paramétrique de détection d'endommagement est proposé, qui suppose seulement que l'on dispose d'enregistrements des vibrations de la structure saine sous différentes températures inconnues, et utilise une opération de moyennisation pour éliminer l'effet de la température.

Mots clés : Identification par sous-espaces, détection d'endommagement, fusion de données délivrées par des capteurs, surveillance vibratoire, structures de génie civil, effet de la température. 


\section{Introduction}

As argued in $[1,2,3]$ and references therein, automatic global vibration-based monitoring techniques have been recognized as useful alternatives to visual inspections or local non destructive evaluations performed manually. It is also widely recognized that the ambient temperature effect on civil engineering structures may result in limitations of such SHM approaches. Actually the dynamics of those structures is affected by the ambient temperature and other environmental effects $[4,5,6]$. Hence both temperature variations and damages affect the modal parameters (modal frequencies, and associated damping values and mode-shapes) which characterize the dynamics of the structure. This raises the issue of discriminating between changes in modal parameters due to damages and changes in modal parameters due to temperature effects.

A parametric damage detection algorithm based on a residual associated with a covariance-driven output-only subspace identification algorithm and a $\chi^{2}$-test built on that residual has been proposed in $[7,8,9,10]$. This subspace-based residual uses the left null space of a particular matrix capturing the nominal (safe) state of the structure. The purpose of this paper is to propose an extension of a non parametric version of this damage detection algorithm, to cope with the temperature effect. This new algorithm only assumes that several datasets are recorded on the safe structure at different and unknown temperatures. The temperature effect is smoothed out using a particular averaging operation.

The paper is organized as follows. In section 2, the subspace-based damaged detection algorithm is summarized, under both its parametric and non parametric versions. In section 3 , the issue of merging multiple measurements setups is addressed, of which the joint handling of measurements recorded at different temperatures is a particular case. A new detection algorithm is proposed which extends the non parametric version. The results obtaining when applying both the original and the new detection algorithms on two application examples are reported in section 4 . The examples are a simulated bridge deck and a laboratory test-case composed of a clamped beam within a climatic chamber. Finally some conclusions are drawn in section 5 .

PI $n^{\circ} 1824$ 


\section{Subspace-based detection}

The subspace-based detection algorithm proposed and investigated in $[7,8,9]$ handles a residual which exploits the left null space of appropriate matrices. This is briefly recalled now.

The use of state-space representations for vibration-based structural monitoring is well known [11, 12]: structural monitoring boils down to monitoring the eigenstructure of the state transition matrix $F$ of a linear dynamic system:

$$
\left\{\begin{aligned}
X_{k+1} & =F X_{k}+V_{k+1} \\
Y_{k} & =H X_{k}
\end{aligned}\right.
$$

namely the pairs $\left(\lambda, \varphi_{\lambda}\right)$ defined by:

$$
\operatorname{det}(F-\lambda I)=0,(F-\lambda I) \varphi_{\lambda}=0, \Phi_{\lambda} \stackrel{\text { def }}{=} H \varphi_{\lambda}
$$

Let

$$
\theta \stackrel{\text { def }}{=}\left(\begin{array}{c}
\Lambda \\
\operatorname{vec} \Phi
\end{array}\right)
$$

be the modal parameter, where $\Lambda$ is the vector whose elements are the $\lambda$ 's, $\Phi$ is the matrix whose columns are the $\Phi_{\lambda}$ 's, and vec denotes the column stacking operator.

The considered residual, which is associated with a covariance-driven outputonly subspace identification algorithm, exploits the following basic although simple factorization property. Let $R_{i} \stackrel{\text { def }}{=}\left(\mathbf{E} Y_{k} Y_{k-i}^{T}\right)$, where $\mathbf{E}$ is the expectation operator, be the output covariance matrix. Let also

$$
\mathcal{H}_{p+1, q} \stackrel{\text { def }}{=}\left(\begin{array}{cccc}
R_{0} & R_{1} & \vdots & R_{q-1} \\
R_{1} & R_{2} & \vdots & R_{q} \\
\vdots & \vdots & \vdots & \vdots \\
R_{p} & R_{p+1} & \vdots & R_{p+q-1}
\end{array}\right) \stackrel{\text { def }}{=} \operatorname{Hank}\left(R_{i}\right)
$$

be the theoretical Hankel matrix. Let also $G \stackrel{\text { def }}{=}\left(\mathbf{E} X_{k} Y_{k}^{T}\right)$ be the cross-covariance between the state and the observed outputs. Direct computations of the $R_{i}$ 's from the Equations in (1) lead to

$$
R_{i}=H F^{i} G
$$

and consequently to the well known factorization:

$$
\mathcal{H}_{p+1, q}=\mathcal{O}_{p+1}(H, F) \mathcal{C}_{q}(F, G)
$$


where $\mathcal{O}_{p}(H, F) \stackrel{\text { def }}{=}\left(\begin{array}{l}H \\ H F \\ \vdots \\ H F^{p-1}\end{array}\right)$ and $\mathcal{C}_{q}(F, G) \stackrel{\text { def }}{=}\left(G F G \cdots F^{q-1} G\right)$ are the observability and controllability matrices, respectively.

From Equation (2), we know that the knowledge of the pair $(H, F)$ is sufficient for recovering the modal parameters. Now it results from Equation (6) that the left factor in the Hankel matrix $\mathcal{H}_{p+1, q}$ depends only on the pair $(H, F)$ and is unaffected by the excitation. Thus the modal parameter $\theta$ can be recovered from matrix $\mathcal{O}_{p}$ only.

\subsection{Parametric test}

The modal parameter vector $\theta$ can be characterized as follows. Assume that the eigenvectors of $F$ are chosen as the basis for the state space of the system in Equation (1). In that basis, matrix $\mathcal{O}_{p+1}$ writes:

$$
\mathcal{O}_{p+1}(\theta)=\left(\begin{array}{l}
\Phi \\
\Phi \Delta \\
\vdots \\
\Phi \Delta^{p}
\end{array}\right)
$$

where diagonal matrix $\Delta=\operatorname{diag}(\Lambda)$, and $\Lambda$ and $\Phi$ are as in Equation (3) above. From Equation (6) it results that whether a nominal modal parameter $\theta_{0}$ is in agreement with a given output covariance sequence $\left(R_{j}\right)_{j}$ is characterized by:

$$
\mathcal{O}_{p+1}\left(\theta_{0}\right) \text { and } \mathcal{H}_{p+1, q} \text { have the same left null space. }
$$

This property can be checked as follows. From $\theta_{0}$, compute $\mathcal{O}_{p+1}\left(\theta_{0}\right)$ using Equation (7). Then perform e.g. a singular value decomposition (SVD) of $\mathcal{O}_{p+1}\left(\theta_{0}\right)$ for defining its left null space, namely for extracting an orthonormal matrix $S$ such that $S^{T} S=I_{s}$ and:

$$
S^{T}\left(\theta_{0}\right) \mathcal{O}_{p+1}\left(\theta_{0}\right)=0
$$

Matrix $S$ depends implicitly on $\theta_{0}$. Although not unique, - two such matrices are related through a post-multiplication with an orthonormal matrix $U$ - it can be treated as a function $S\left(\theta_{0}\right)$ [7]. Then the characteristic property in Equation (8) writes:

$$
S^{T}\left(\theta_{0}\right) \mathcal{H}_{p+1, q}=0
$$

PI $n^{\circ} 1824$ 
Now, the damage detection problem is to decide whether a new data sample $\left(Y_{k}\right)_{k=1, \ldots, n}$ from the (possibly damaged) system is still well described by a reference parameter $\theta_{0}$ (identified on data recorded on the undamaged reference system) or not. Let $\widehat{\mathcal{H}}_{p+1, q}$ be the empirical counterpart of Equation (4) computed with the new data:

$$
\widehat{\mathcal{H}}_{p+1, q} \stackrel{\text { def }}{=} \operatorname{Hank}\left(\widehat{R}_{i}\right), \quad \widehat{R}_{i}=\frac{1}{n} \sum_{k=1}^{n} Y_{k} Y_{k-i}^{T}
$$

For checking whether the new data agree with the reference modal parameter $\theta_{0}$, the following residual is introduced in $[7,10]$ :

$$
\zeta_{n}\left(\theta_{0}\right) \stackrel{\text { def }}{=} \sqrt{n} \operatorname{vec}\left(S^{T}\left(\theta_{0}\right) \widehat{\mathcal{H}}_{p+1, q}\right)
$$

From (10), it can be deduced that $\zeta_{n}\left(\theta_{0}\right)$ has zero mean when no change occurs in $\theta$, and nonzero mean if a change occurs. Thus it plays the role of a residual.

For deciding that the residual $\zeta_{n}\left(\theta_{0}\right)$ is significantly different from zero, a particular asymptotic statistical framework is assumed where the changes to occur are supposed to be small [7]. Under convenient assumptions, the residual is asymptotically Gaussian, and manifests itself the damage by a change in its own mean value. In other words, let $\mathcal{J}\left(\theta_{0}\right)$ and $\Sigma\left(\theta_{0}\right)$ the residual sensitivity and covariance matrices, respectively. Then, provided that $\Sigma\left(\theta_{0}\right)$ is positive definite:

$$
\zeta_{n}\left(\theta_{0}\right) \underset{n \rightarrow \infty}{\longrightarrow}\left\{\begin{array}{cl}
\mathcal{N}\left(0, \Sigma\left(\theta_{0}\right)\right) & \text { in case of no change } \\
\mathcal{N}\left(\mathcal{J}\left(\theta_{0}\right) \delta \theta, \Sigma\left(\theta_{0}\right)\right) & \text { in case of a small deviation } \delta \theta \text { in } \theta
\end{array}\right.
$$

The generalized likelihood ratio (GLR) test, resulting from the maximization w.r.t. $\delta \theta$ of the ratio of the two Gaussian distributions in (13), provides us with the following $\chi^{2}$-test statistics built on the residual (12):

$\chi_{n}^{2}\left(\theta_{0}\right) \stackrel{\text { def }}{=} \zeta_{n}^{T}\left(\theta_{0}\right) \widehat{\Sigma}^{-1}\left(\theta_{0}\right) \widehat{\mathcal{J}}\left(\theta_{0}\right)\left(\widehat{\mathcal{J}}^{T}\left(\theta_{0}\right) \widehat{\Sigma}^{-1}\left(\theta_{0}\right) \widehat{\mathcal{J}}\left(\theta_{0}\right)\right)^{-1} \widehat{\mathcal{J}}^{T}\left(\theta_{0}\right) \widehat{\Sigma}^{-1}\left(\theta_{0}\right) \zeta_{n}\left(\theta_{0}\right)$

which should be compared to a threshold. In Equation (14), $\widehat{\mathcal{J}}\left(\theta_{0}\right)$ and $\widehat{\Sigma}\left(\theta_{0}\right)$ are consistent estimates of the sensitivity and covariance matrices of $\zeta_{n}\left(\theta_{0}\right)$ [7].

\subsection{Non-parametric test}

In some cases, it may be of interest to replace this parametric approach with a non parametric one, based on an empirical null space computed on a reference data 
set and not on a reference modal signature. Such a null space may result from a SVD of the empirical Hankel matrix built on the reference data set (indexed with superscript 0$)$ :

$$
\widehat{S}_{0}^{T} \widehat{\mathcal{H}}_{p+1, q}^{(0)}=0
$$

Such a non parametric detection approach is used e.g. in $[13,14]$.

Based on new data from the (possibly damaged) system, the empirical Hankel matrix $\widehat{\mathcal{H}}_{p+1, q}$ is computed and the residual then writes:

$$
\widehat{\zeta}_{n} \stackrel{\text { def }}{=} \sqrt{n} \operatorname{vec}\left(\widehat{S}_{0}^{T} \widehat{\mathcal{H}}_{p+1, q}\right)
$$

Let $\Sigma$ be the covariance matrix of $\widehat{\zeta}$. Then, provided that $\Sigma$ is positive definite:

$$
\widehat{\zeta}_{n} \underset{n \rightarrow \infty}{\longrightarrow}\left\{\begin{aligned}
\mathcal{N}(0, \Sigma) & \text { in case of no change } \\
\mathcal{N}(\delta \Upsilon, \Sigma) & \text { in case of a small deviation } \delta \Upsilon \text { in } \widehat{\zeta}
\end{aligned}\right.
$$

The GLR test, resulting from the maximization w.r.t. $\delta \Upsilon$ of the ratio of the two Gaussian distributions in (17), provides us with the following $\chi^{2}$-test statistics built on the residual (16):

$$
\widehat{\chi}_{n}^{2} \stackrel{\text { def }}{=} \widehat{\zeta}_{n}^{T} \widehat{\Sigma}^{-1} \widehat{\zeta}_{n}
$$

which should also be compared to a threshold. In Equation (18), $\widehat{\Sigma}$ is a consistent estimate of the covariance matrix of $\widehat{\zeta}_{n}$.

\section{$3 \quad$ Merging multiple measurements setups}

It has been shown both experimentally and theoretically that covariance-driven subspace identification algorithm tend to factor out the non stationarities in the excitation, thanks to the averaging operations in Equation (11) and the key factorization property in Equation (6) $[15,16,17]$. The Hankel matrix factorization property in Equation (6) has also proven key to the extension of that algorithm to the simultaneous processing of multiple measurements setups recorded at different times and under different excitation conditions $[18,17]$. When the sensors setups are all identical (same number and locations of sensors), this extended algorithm boils down to process the empirical average of the individual Hankel matrices associated with each sensor setup.

Hence, when $J$ reference data sets are available, as e.g. when handling the temperature effect, a global empirical Hankel matrix is computed by averaging the

PI $n^{\circ} 1824$ 
empirical Hankel matrices corresponding to each reference data set:

$$
\overline{\mathcal{H}}_{p+1, q}^{(0)} \stackrel{\text { def }}{=} \frac{1}{J} \sum_{j=1}^{J} \overline{\mathcal{H}}_{p+1, q}^{(0), j}
$$

Using a SVD as above, a global empirical null space $\bar{S}_{0}$ can then be obtained:

$$
\bar{S}_{0}^{T} \overline{\mathcal{H}}_{p+1, q}^{(0)}=0
$$

For handling the temperature effect, the idea proposed in this paper consists in assuming that $J$ reference data sets are available for the undamaged structure at different unknown temperatures ${ }^{1}$, and in using the trick in Equations (19)-(20) as the entry for the non parametric subspace-based detection algorithm in Equations (16)-(18). It should be clear, however, that the situation with those $J$ data sets is basically different from that of reference data sets recorded for the same structure subject to different excitations. Here, because of the temperature effect on the dynamics of the structure, the reference data sets actually correspond to different states of the undamaged structure.

The proposed algorithm runs as follows. Compute the global empirical null space $\bar{S}_{0}$ in Equation (20) corresponding to the $J$ reference data sets at the different temperatures. Based on new data from the (possibly damaged) system, fill the empirical Hankel matrix $\widehat{\mathcal{H}}_{p+1, q}$ and compute the residual:

$$
\bar{\zeta}_{n} \stackrel{\text { def }}{=} \sqrt{n} \operatorname{vec}\left(\bar{S}_{0}^{T} \widehat{\mathcal{H}}_{p+1, q}\right)
$$

With $\bar{\Sigma}$ an estimate of the covariance matrix of $\bar{\zeta}$, the corresponding $\chi^{2}$-test statistics writes:

$$
\bar{\chi}_{n}^{2} \stackrel{\text { def }}{=} \bar{\zeta}_{n}^{T} \bar{\Sigma}^{-1} \bar{\zeta}_{n}
$$

This damage detection algorithm is expected to smooth out the temperature effect thanks to the averaging operation in Equation (19).

\section{Application}

Both algorithms in Equations (14) and (22) have been experimented on two cases: a simulated bridge deck provided by LMSSMat and a vertical clamped beam within a climatic chamber, a test-case provided by LCPC.

\footnotetext{
${ }^{1}$ A similar idea is used in $[19,20]$.
} 


\subsection{Simulation: simple bridge deck}

In this section, a simulated bridge deck is described, with controlled temperature variations. The temperature effect on the modal parameters and on the test results is discussed.

\subsubsection{The test-case}

In order to outline the temperature effect and to test the validity of the proposed damage detection algorithm with temperature rejection, a simulated bridge deck has been simulated.

This simplified bridge model consists of 28 supports and a deck which is $3 \mathrm{~m}$ high, 6.6 to $10 \mathrm{~m}$ wide, and $100 \mathrm{~m}$ long, as shown in Figure 1 (Left). The Structural Dynamics Toolbox [21] was used to develop and implement the finite element model of this bridge. The whole bridge span is modeled using 9600 standard 8-node isoparametric volume elements and 13668 nodes. At both ends, the span motion is blocked in the $y$ and $z$ directions, while a nominal stiffness of $1 e 10 \mathrm{~N} / \mathrm{m}$ is used, in the bridge $x$ direction, to simulate a blocking condition.

The temperature variations are modeled using either one of two fields: a uniform temperature elevation and a linear variation with $z$ from $25^{\circ} \mathrm{C}$ on the deck to $15^{\circ} \mathrm{C}$ at the bottom. The vertical thermal gradient field and the induced axial strain $\sigma_{x x}$ state are depicted on Figure 2. The warmer deck tends to expand while the cooler bottom contracts. The figure also clearly shows edge effects where the springs are used to model attachment conditions near the piles.

The damage is simulated as the reduction in the stiffness of some elements. More precisely, the damaged elements, whose location is $16.5 \mathrm{~m}$ from the end, are modeled as a reduction of the material modulus by up to $30 \%$. The damaged area consists of two sections, each of which includes 48 elements. But, as displayed in Figure 1 (Right), only 44 of those elements are simulated as damaged, so there are 88 damaged elements altogether within the bridge model. Figure 1 also shows the location of the simulated damaged sections and elements in the bridge.

Simulation scenarios for both the undamaged case, which serves as the reference signal for subsequent damage detection, and the damaged case are considered. For each case, 21 temperature scenarios are undertaken, and each scenario is repeated 50 times. The output data are simulated under white noise excitations. 21 sensors are uniformly distributed along the bridge to measure the vertical displacement, and the sensors location is also shown in Figure 1. The sampling frequencies for all sensors are $256 \mathrm{~Hz}$ and 100000 sample points are recorded for each sensor.

PI $n^{\circ} 1824$ 
Figure 3 displays how the first frequency is affected by a uniform temperature decrease, under safe and damaged conditions, and how the corresponding partial $\chi^{2}$-test focussed on that frequency is affected.

\subsubsection{Numerical results}

The reference parameter $\theta_{0}$, the null space $S\left(\theta_{0}\right)$ in Equation (9) and matrices $\widehat{\mathcal{J}}\left(\theta_{0}\right), \widehat{\Sigma}\left(\theta_{0}\right)$ for the test in Equations (12)-(14) are estimated on the first scenario where there is no pre-stress. The global null space $\bar{S}_{0}$ in Equation (20) and matrix $\bar{\Sigma}$ for the non parametric test in Equations (21)-(22) are estimated are estimated on the whole set of 10 realizations of the scenarios for the undamaged case.

On Figures 4-5, the values of the original subspace-based $\chi^{2}$-test in Equation (14) for the 21 scenarios are displayed on the left hand side, and the values of the new $\chi^{2}$-test in Equation (22) are displayed on the right hand side. On Figure 4 are displayed all the test values obtained for the 10 repetitions of the 21 scenarios. These results are condensed in Figure 5 where each test value is the empirical mean of 10 realizations of the same scenario.

On Figure 4 (Left), it is possible to discriminate between the damaged and the undamaged values for the test in Equation (14). Whereas on Figure 4 (Right), it is possible to set a threshold for separating the values of the new test for the damaged and safe scenarii. Figure 5 (Right) provides us with the same information about the dispersion of the test values, which are much higher in the damaged case, as theoretically predicted. These results show that, for the present experiment, either the mean test value in Figure 5 (Right) or any single test value in Figure 4 (Right) is sufficient to detect the damage when the temperature $T$ varies.

\subsection{Laboratory test-case: clamped beam within a climatic chamber}

\subsubsection{The test-case}

The experimental device is depicted in Figure 6. A vertical beam is clamped at both ends on a workbench made of four vertical thick columns and two horizontal decks. This workbench is made of steel, whereas the beam is made of aluminum. The whole apparatus is set inside a climatic chamber with controlled ambient temperature. Because steel and aluminum have different thermal expansion coefficients (11.7e -6 and $23.4 e-6 K^{-1}$, respectively), a temperature change naturally induces a significant axial prestress inside the beam, constant along the beam in the absence of external forces. 
The test beam is instrumented with four accelerometers, located at nodes of the 5th flexural mode in order to avoid nodes of modes 1 to 4 (a truncation up to the 4th mode has been performed for the analysis reported in [22]). A pair of aluminum strain gauges with thermal compensation is also bonded on the beam. The half sum of both strains gives a direct measure of the actual axial prestress (divided by Young's modulus), without requiring any temperature measurement. This measurement, not useful for the present detection method, it is of primary importance for the method in [22]. Some temperature sensors have also been used in order to check that gage measurements are closely related to thermal variations.

Tests are carried out inside the climatic chamber, first by stabilizing the ambient temperature for 1 hour, and then cooling down for 17 hours with a slope of $-1{ }^{\circ} \mathrm{C}$ per hour. The beam is acoustically excited by a loudspeaker with a white noise input. Strains and temperatures measurements are saved every seconds. Acceleration measurements are automatically triggered every 30 minutes, for 600 seconds with sampling frequency $1280 \mathrm{~Hz}$, which is sufficient for modes 1 to 4 (the 4 th frequency is below $500 \mathrm{~Hz}$ ).

An example of experimental results is given in Figure 7 (undamaged case). It clearly shows that prestress, and thus frequencies, increase as temperature decreases with time (note that prestress is taken positive when tensile). At the end of the test, the first frequency has been increased by about 15\%. Modes 2, 3 and 4 (not shown here) have been increased by about 8,5 and $3 \%$, respectively. Axial prestress has thus a stronger effect for lower frequencies, which is coherent with known results [23].

Finally, two sets of experiments are performed, for the safe and damaged cases respectively. In each set, 37 temperature scenarios are undertaken, and each scenario is repeated 10 times (one scenario every 30 minutes). The effect of an horizontal spring attached to the beam plays the role of a local non destructive damage. This damage may be tuned by increasing the stiffness and/or the height of the spring. In this experiment, the spring is located at $L / 5$ and its stiffness is about $k=4000 \mathrm{~N} \cdot \mathrm{m}^{-1}$. This choice has been designed from a finite element model in order to give slight (less than 1\%) deviations from the no-spring "safe" case. Experimental deviations of modes 1 to 4 are about $+0.8,+0.4,-0.1$ and $-0.2 \%$, respectively. Negative deviations for modes 3 and 4 are a bit odd, but may result from a mechanical coupling of the beam with the workbench and the spring bracket. Figure 8 shows how the first frequencies are affected by the temperature scenarios, under both safe (blue) and damaged (red) conditions.

PI $n^{\circ} 1824$ 


\subsubsection{Numerical results}

The reference parameter $\theta_{0}$, the null space $S\left(\theta_{0}\right)$ in Equation (9) and matrices $\widehat{\mathcal{J}}\left(\theta_{0}\right), \widehat{\Sigma}\left(\theta_{0}\right)$ for the test in Equations $(12)-(14)$ are estimated on the 10 realizations of the first scenario corresponding to a thermal constraint about 20 . The global null space $\bar{S}_{0}$ in Equation (20) and matrix $\bar{\Sigma}$ for the non parametric test in Equations (21)-(22) are estimated on the whole set of 10 realizations of the scenarios for the undamaged case.

In Figures 9-10, the values of the original subspace-based $\chi^{2}$-test in Equation (14) for the 25 scenarios corresponding to thermal constraints $\varepsilon_{x}-\alpha \delta T$ ranging approximately between 20 and 115, are displayed on the left hand side, and the values of the new $\chi^{2}$-test in Equation (22) are displayed on the right hand side. On Figure 9 are displayed all the test values obtained for the 10 repetitions of the 25 scenarii. These results are condensed in Figure 10 where each test value is the empirical mean of 10 realizations of the same scenario. It should be obvious that 10 is too small a number for clearing out possible outliers; this is confirmed on Figure 10 (Right).

On Figure 9 (Left), it is not possible to discriminate between the damaged and the undamaged values for test in Equation (14). Whereas on Figure 9 (Right), it is possible to set a threshold for separating the test values of the damaged and safe scenarii. Figure 10 (Right) provides us with the same information about the dispersion of the test values, which are much higher in the damaged case, as theoretically predicted. These results also show that, for the present experiment, either the mean test value in Figure 10 (Right) or any single test value in Figure 9 (Right) is sufficient to detect the damage when the temperature $T$ varies.

\section{Conclusion}

The problem of handling the temperature effect in vibration-based monitoring of civil engineering structures has been addressed. A non parametric approach has been proposed, based on a global empirical null space merging reference data sets recorded at unknown temperatures, and plugged into a statistical subspace damage detection algorithm. The relevance of the proposed algorithm has been illustrated on two examples : a simulated bridge deck model and a laboratory test-case made of a clamped beam within a climatic chamber.

Issues for future research encompass validation on structures in-operation, and extension to the non stationary excitation case. Actually, computing the test co- 
variance matrix for each scenario, as performed in [22], would increase if not ensure the robustness of the approach w.r.t. changes in the excitation.

\section{References}

[1] Farrar, C.R., Doebling, S.W. and Nix, D.A. (2001). Vibration-based structural damage identification. Philosophical Transactions of the Royal Society: Mathematical, Physical and Engineering Sciences, 359(1778): 131-150.

[2] Natke, H.G. and Cempel, C. (1997). Model-Aided Diagnosis of Mechanical Systems: Fundamentals, Detection, Localization, Assessment, Springer-Verlag.

[3] Fritzen, C.-P. (2005). Recent developments in vibration-based structural health monitoring. Proceedings of the 5th International Workshop on Structural Health Monitoring, Stanford, CA., Sep.12-14, 2005, 42-60.

[4] Peeters, B., J. Maeck and G. de Roeck (2001). Vibration based damage detection in civil engineering: excitation sources and temperature effects. Smart Materials and Structures 10(3), 518-527.

[5] Rohrmann, R.G., M. Baessler, S. Said, W. Schmid and W.F. Ruecker (2000). Structural causes of temperature affected modal data of civil structures obtained by long time monitoring. Proceedings of the 18th International Modal Analysis Conference, San Antonio, TX, Feb.7-10, 2000.

[6] Kullaa, J. (2004). Structural health monitoring under variable environmental or operational conditions. Proceedings of the 2nd European Workshop on Structural Health Monitoring, Munich, FRG, Jul. 7-9, 2004, 1262-1269.

[7] Basseville, M., Abdelghani, M. and Benveniste, A. (2000). Subspace-based fault detection algorithms for vibration monitoring. Automatica, 36(1): 101-109.

[8] Mevel, L., Hermans, L. and Van der Auweraer, H. (1999). Application of a subspace-based fault detection method to industrial structures. Mechanical Systems and Signal Processing, 13(6): 823-838.

[9] Basseville, M., Mevel, L., Vechio, A., Peeters, B. and Van der Auweraer, H. (2003). Output-only subspace-based damage detection - Application to a reticular structure. Structural Health Monitoring, 2(2): 161-168.

PI $n^{\circ} 1824$ 
[10] Basseville, M., Mevel, L. and Goursat, M. (2004). Statistical model-based damage detection and localization: subspace-based residuals and damage-to-noise sensitivity ratios. Journal of Sound and Vibration 275(3-5), 769-794.

[11] Juang, J.N. (1994). Applied System Identification. Prentice Hall, Englewood Cliffs, NJ.

[12] Ewins, D.J. (2000). Modal Testing: Theory, Practice and Applications (2nd ed.). Research Studies Press, Letchworth, Hertfordshire.

[13] Fritzen, C.-P. and Mengelkamp, G. (2002). Detection of delaminations in composite materials using a smart structures concept. Proceedings of the 1st European Workshop on Structural Health Monitoring, Paris, F., July 10-12, 2002, 237-244.

[14] Yan, A.-M. and Golinval, J.-C. (2006). Null subspace-based damage detection of structures using vibration measurements. Mechanical Systems and Signal Processing, 20(3), 611-626.

[15] Benveniste, A. and Fuchs, J.J. (1985). Single sample modal identification of a non-stationary stochastic process. IEEE Transactions on Automatic Control, 30(1), 66-74.

[16] Benveniste, A. and Mevel, L. (2007). Nonstationary consistency of subspace methods. IEEE Transactions on Automatic Control, 52, to appear.

[17] Mevel, L., Benveniste, A., Basseville, M. and Goursat, M. (2002). Blind subspace-based eigenstructure identification under nonstationary excitation using moving sensors. IEEE Transactions on Signal Processing, 50(1), 41-48,

[18] Mevel, L., Basseville, M., Benveniste, A. and Goursat, M. (2002). Merging sensor data from multiple measurement setups for nonstationary subspace-based modal analysis. Journal of Sound and Vibration, 249(4), 719-741.

[19] Yan, A.-M., Kerschen, G., De Boe, P. and Golinval, J.-C. (2005a). Structural damage diagnosis under varying environmental conditions - Part I: A linear analysis. Mechanical Systems and Signal Processing, 19(4), 847-864.

[20] Yan, A.-M., Kerschen, G., De Boe, P. and Golinval, J.-C. (2005b). Structural damage diagnosis under varying environmental conditions - Part II: Local PCA for non-linear cases. Mechanical Systems and Signal Processing, 19(4), 865-880. 
[21] Balmès, E. and Leclère, J.-M. (2006). Structural Dynamics Toolbox. User's Guide Version 5.3, SD Tools, Paris, F.

[22] Basseville, M., Bourquin, F., Mevel, L., Nasser, H. and Treyssède, F. (2006). Handling the temperature effect in SHM: combining a subspace-based statistical test and a temperature-adjusted null space. Proceedings of the 3rd European Workshop on Structural Health Monitoring, Granada, S., July 5-7, 2006, 759766.

[23] Bokaian, A. (1988). Natural frequencies of beams under compressive axial loads. Journal of Sound and Vibration, 126(1), 49-65.

\section{List of Figures}

1 Bridge deck - Top: The model with the sensors location. Bottom: The damaged area. . . . . . . . . . . . . . . . . . 16

2 Bridge deck - Top: Linear thermal field. Bottom: Induced axial stress. . . . . . . . . . . . . . . . . . 17

3 Bridge deck - Decreasing temperature effect. Left: On the first frequency. Right: On the partial $\chi^{2}$-test monitoring the first frequency. Safe (triangle), damaged (diamond) . . . . . . . . . . . .

4 Bridge deck - Test values. Safe (blue) and damaged (red) cases. $X$-axis: Temperature variation. $Y$-axis: Test values. Left: Original $\chi^{2}$-test in Equation (14). Right: New $\chi^{2}$-test in Equation (22). . . . 18

$5 \quad$ Bridge deck - Test values averaged over the 10 experiments in Figure 4. 18

$6 \quad$ Beam - Workbench (left) and instrumented test beam (right). . . . 19

7 Beam - Axial prestress and 1st frequency vs time (left), temperature (right) . . . . . . . . . . . . . . . . . . . . 19

8 Beam - Temperature effect on the second (left), third (middle) and fourth (right) frequencies. Safe (blue) and damaged (red) cases. $X$ axis: Thermal constraint. $Y$-axis: Frequencies. . . . . . . . . .

$9 \quad$ Beam - Test values. Safe (blue) and damaged (red) cases. $X$-axis: Thermal constraint. $Y$-axis: Test values. Left: Original $\chi^{2}$-test in Equation (14). Right: New $\chi^{2}$-test in Equation (22). . . . . . . . 20

10 Beam - Test values averaged over the 10 experiments in Figure 9. . 20 

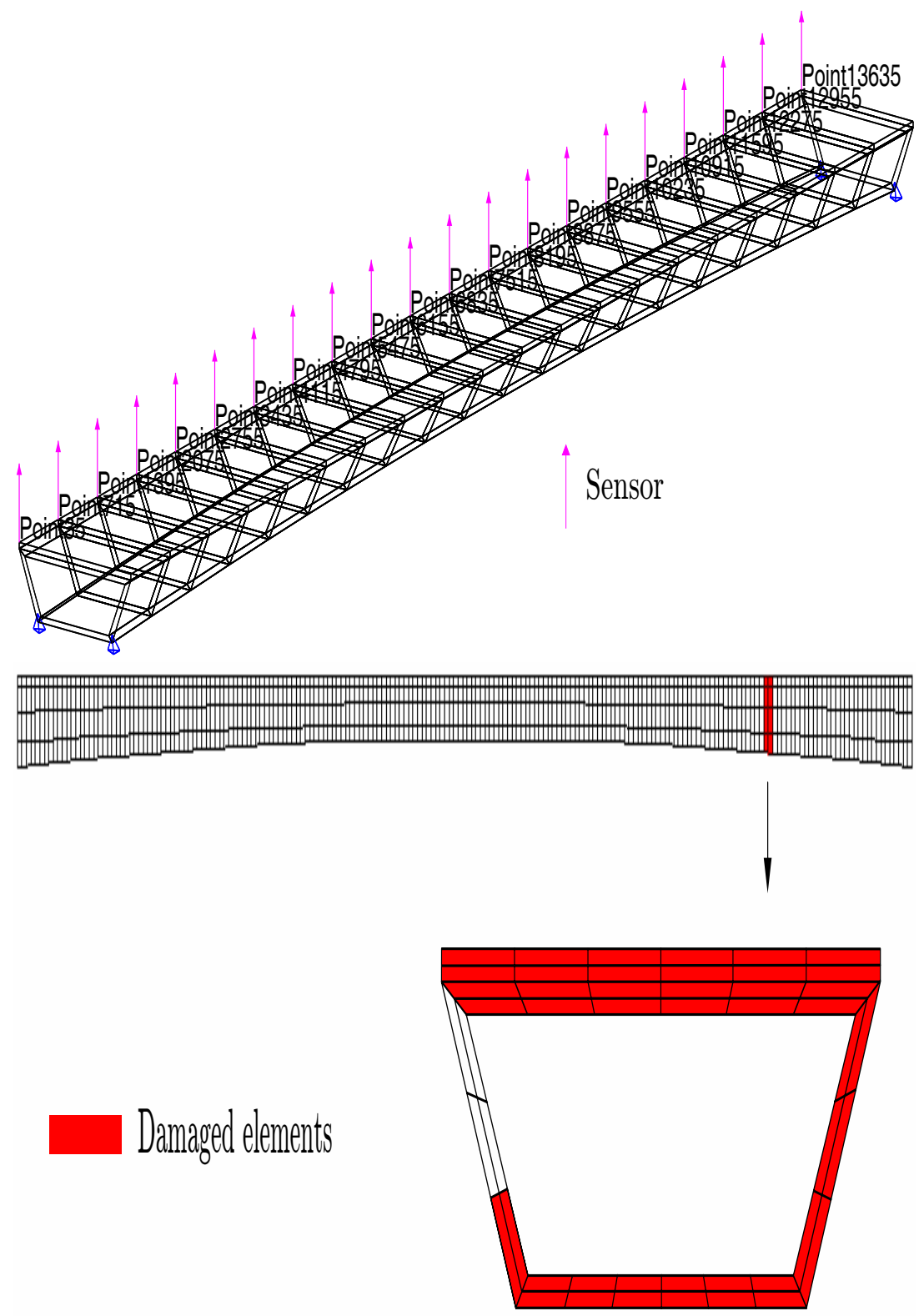

Figure 1: Bridge deck - Top: The model with the sensors location. Bottom: The damaged area. 


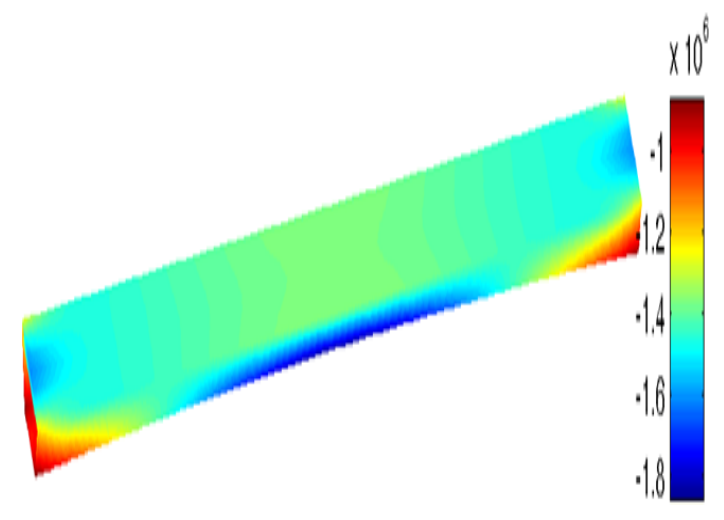

Figure 2: Bridge deck - Top: Linear thermal field. Bottom: Induced axial stress.

PI $n^{\circ} 1824$ 

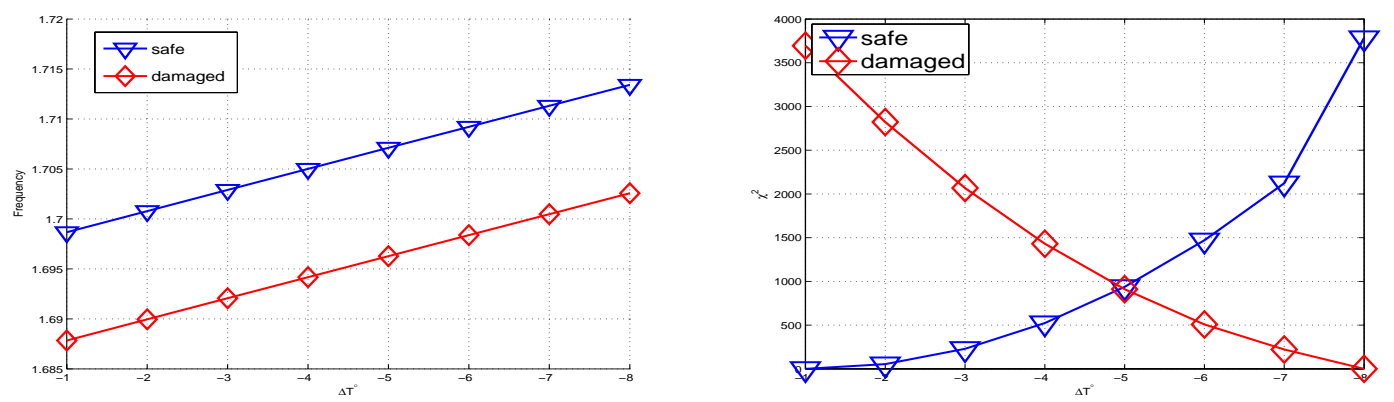

Figure 3: Bridge deck - Decreasing temperature effect. Left: On the first frequency. Right: On the partial $\chi^{2}$-test monitoring the first frequency. Safe (triangle), damaged (diamond).
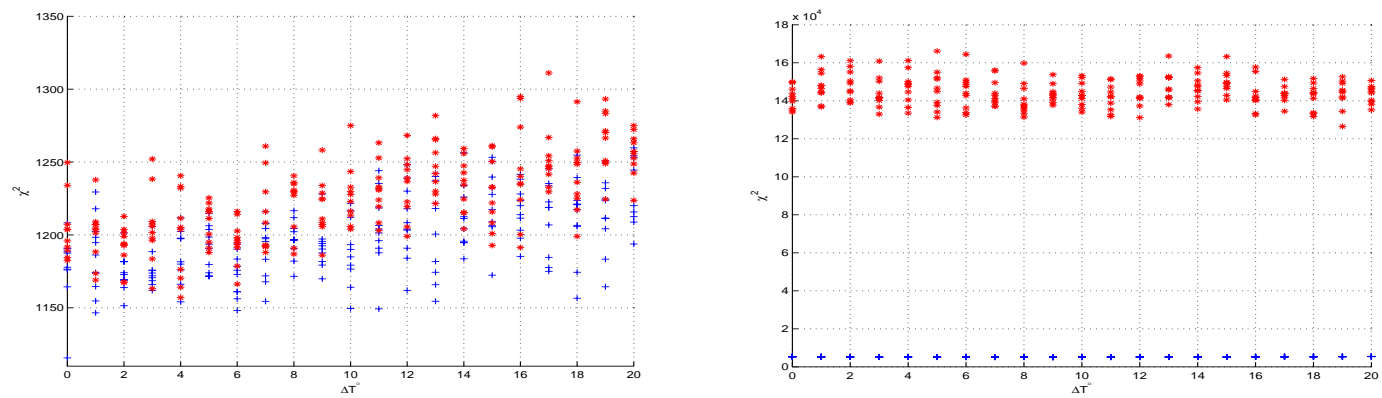

Figure 4: Bridge deck - Test values. Safe (blue) and damaged (red) cases. $X$-axis: Temperature variation. $Y$-axis: Test values. Left: Original $\chi^{2}$-test in Equation (14). Right: New $\chi^{2}$-test in Equation (22).
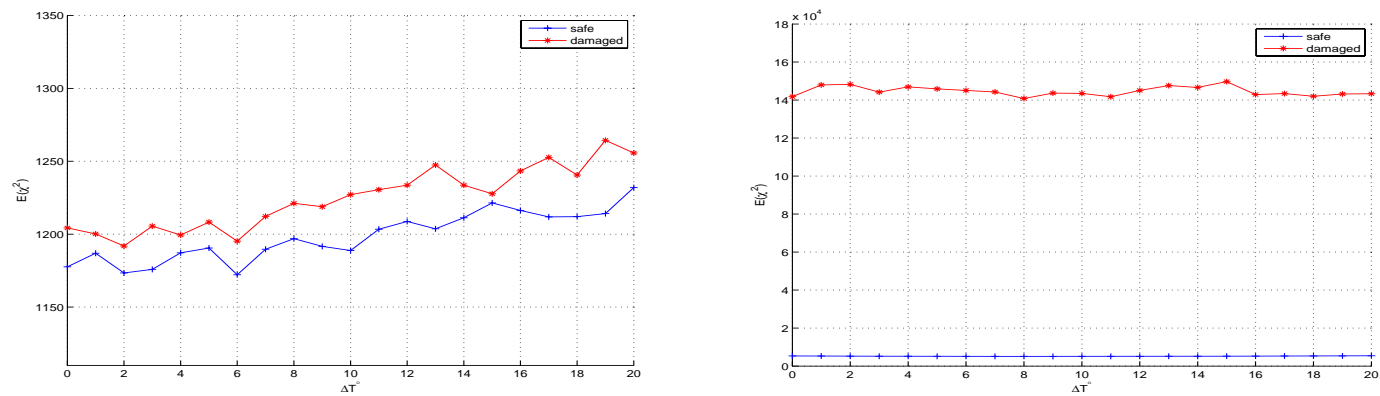

Figure 5: Bridge deck - Test values averaged over the 10 experiments in Figure 4. 

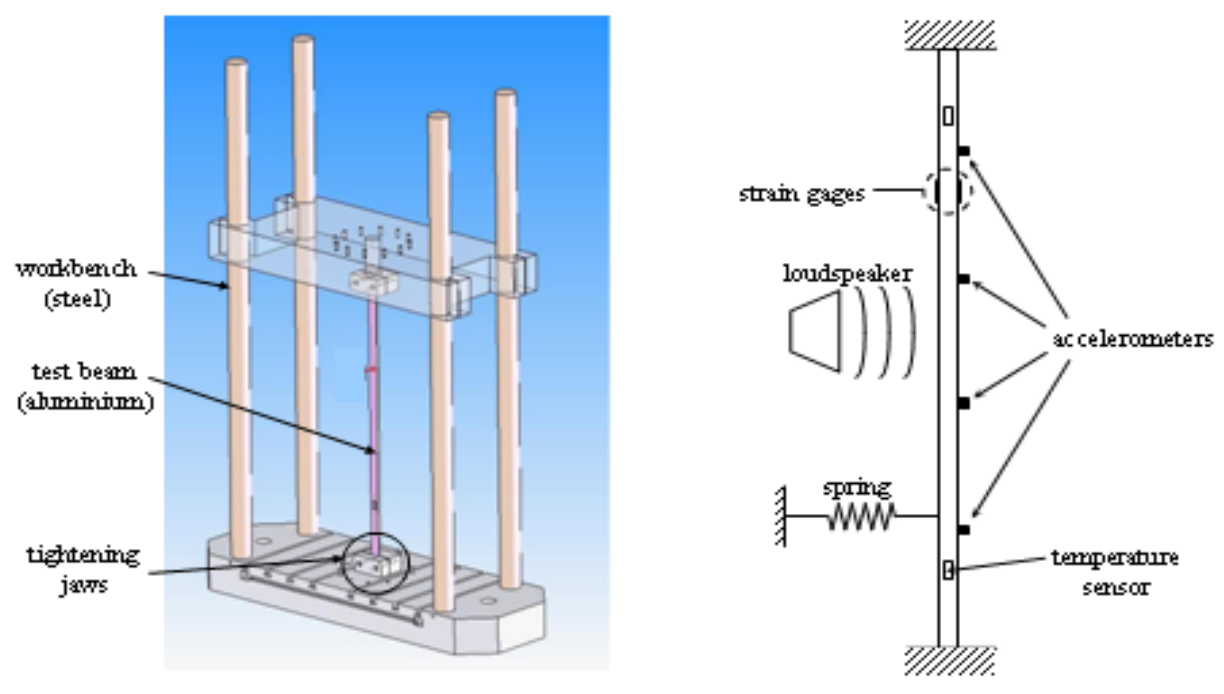

Figure 6: Beam - Workbench (left) and instrumented test beam (right).
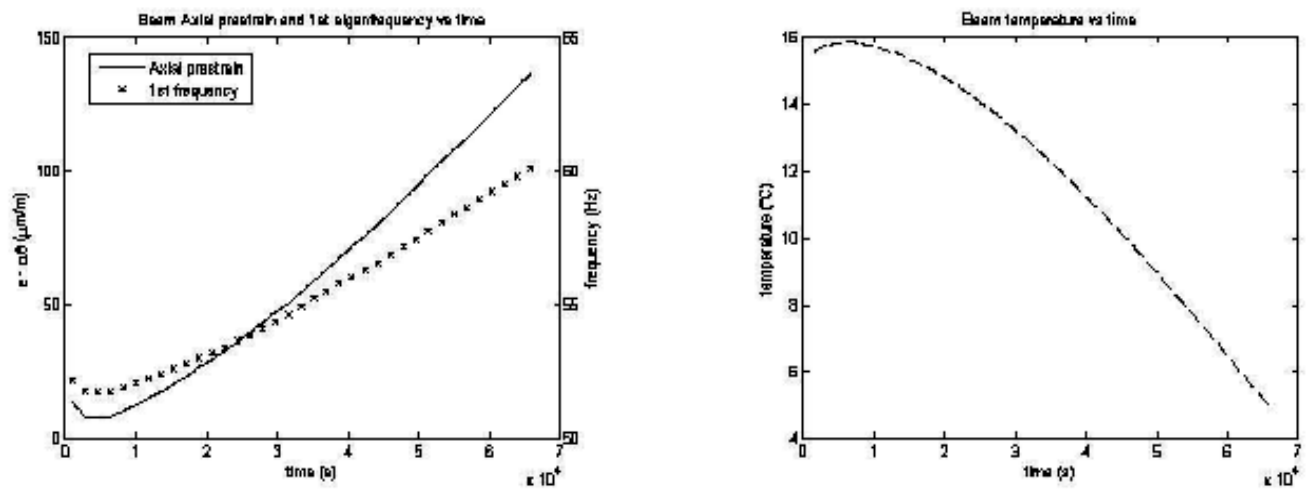

Figure 7: Beam - Axial prestress and 1st frequency vs time (left), temperature (right).

PI n ${ }^{\circ} 1824$ 

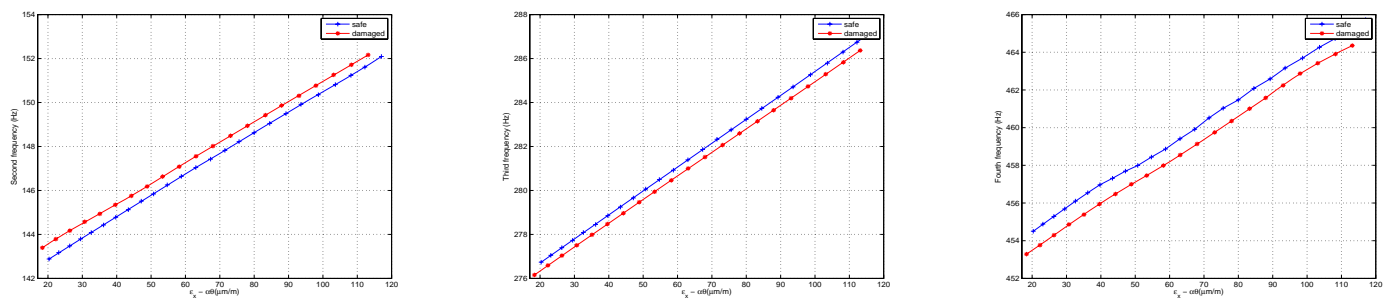

Figure 8: Beam - Temperature effect on the second (left), third (middle) and fourth (right) frequencies. Safe (blue) and damaged (red) cases. $X$-axis: Thermal constraint. $Y$-axis: Frequencies.
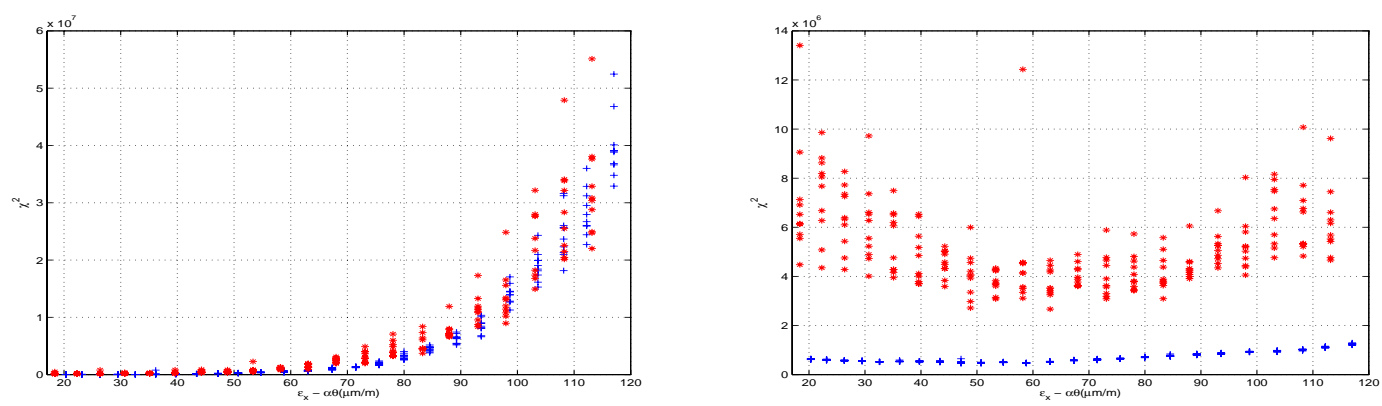

Figure 9: Beam - Test values. Safe (blue) and damaged (red) cases. $X$-axis: Thermal constraint. $Y$-axis: Test values. Left: Original $\chi^{2}$-test in Equation (14). Right: New $\chi^{2}$-test in Equation (22).
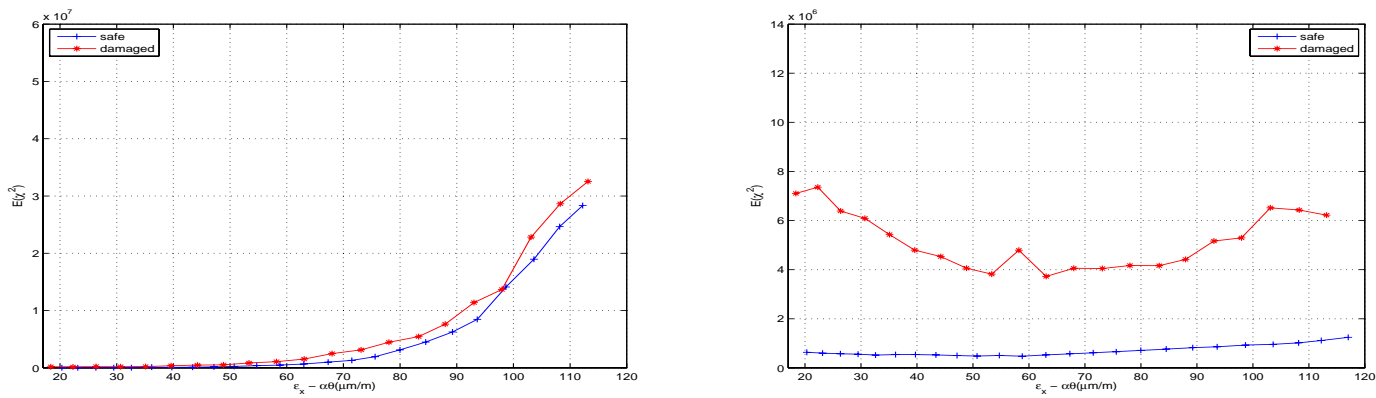

Figure 10: Beam - Test values averaged over the 10 experiments in Figure 9. 


\section{Contents}

1 Introduction $\quad 3$

2 Subspace-based detection 4

2.1 Parametric test ...................... 5

2.2 Non-parametric test .................. 6

3 Merging multiple measurements setups $\quad 7$

4 Application $\quad 8$

4.1 Simulation: simple bridge deck . . . . . . . . . . . . . . . 9

4.1 .1 The test-case . . . . . . . . . . . . . . 9

4.1 .2 Numerical results . . . . . . . . . . . . . . . . . . . 10

4.2 Laboratory test-case: clamped beam within a climatic chamber . . . 10

4.2 .1 The test-case . . . . . . . . . . . . . . 10

4.2 .2 Numerical results . . . . . . . . . . . . . . . . . 12

5 Conclusion $\quad 12$

PI n ${ }^{\circ} 1824$ 